\title{
Correlation between GenoType MTBDRplus Assay and Phenotypic Susceptibility Test for Prothionamide in Patients with Genotypic Isoniazid Resistance
}

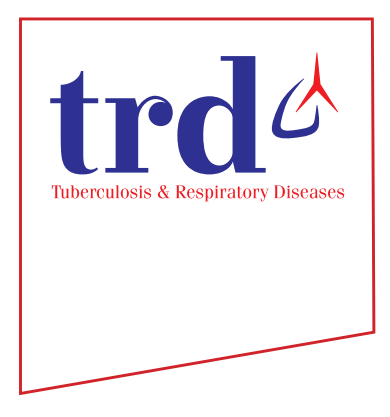

\author{
Joo Hee Lee, M.D. ${ }^{\mathbb{D}}$, Kyung-Wook Jo, M.D., Ph.D. and Tae Sun Shim, M.D., Ph.D. (iD \\ Department of Pulmonary and Critical Care Medicine, Asan Medical Center, University of Ulsan College of Medicine, Seoul, Korea
}

Background: The purpose of this study was to analyze the relationship between the gene mutation patterns by the GenoType MTBDRplus (MTBDRplus) assay and the phenotypic drug susceptibility test (pDST) results of isoniazid (INH) and prothionamide (Pto).

Methods: A total of 206 patients whose MTBDRplus assay results revealed $k a t G$ or $i n h A$ mutations were enrolled in the study. The pDST results were compared to mutation patterns on the MTBDRplus assay.

Results: The $k a t G$ and $i n h A$ mutations were identified in $68.0 \%$ and $35.0 \%$ of patients, respectively. Among the 134 isolated $\mathrm{katG}$ mutations, three (2.2\%), 127 (94.8\%) and 11 (8.2\%) were phenotypically resistant to low-level INH, highlevel INH, and Pto, respectively. Among the 66 isolated inhA mutations, 34 (51.5\%), 18 (27.3\%) and 21 (31.8\%) were phenotypically resistant to low-level INH, high-level INH, and Pto, respectively. Of the 34 phenotypic Pto resistant isolates, 21 (61.8\%), 11 (32.4\%), and two (5.9\%) had inhA, katG, and both gene mutations.

Conclusion: It is noted that Pto may still be selected as one of the appropriate multidrug-resistant tuberculosis regimen, although inhA mutation is detected by the MTBDRplus assay until pDST confirms a Pto resistance. The reporting of detailed mutation patterns of the MTBDRplus assay may be important for clinical practice, rather than simply presenting resistance or susceptibility test results.

Keywords: katG Protein; InhA Protein; Isoniazid; Prothionamide; Genotype; Biological Assay; Disease Susceptibility; Research Design; Mycobacterium tuberculosis; Mycobacterium

Address for correspondence: Tae Sun Shim, M.D., Ph.D.

Department of Pulmonary and Critical Care Medicine, Asan Medical

Center, University of Ulsan College of Medicine, 88 Olympic-ro 43-gil,

Songpa-gu, Seoul 05505, Korea

Phone: 82-2-3010-3892, Fax: 82-2-3010-6968

E-mail: shimts@amc.seoul.kr

Received: Mar. 28, 2018

Revised: Apr. 28, 2018

Accepted: Jun. 25, 2018

Published online: Sep. 28, 2018

(c) It is identical to the Creative Commons Attribution Non-Commercial License (http://creativecommons.org/licenses/by-nc/4.0/).

The Korean Academy of Tuberculosis and Respiratory Diseases.

\section{Introduction}

Tuberculosis (TB) is a global health problem. According to the World Health Organization (WHO) report, there were an estimated 10.4 million new TB cases worldwide in 2015, of which 480,000 cases were multidrug-resistant TB (MDR-TB), and an additional 100,000 cases were rifampin (RIF)-resistant TB that were also newly eligible for MDR-TB treatment ${ }^{1}$. In Korea, isoniazid (INH) resistance rates were $9.9 \%$ in new patients and $24.1 \%$ in retreatment patients ${ }^{2}$. The rate of MDR-TB among new TB cases increased from $2.7 \%$ in 2004 to $2.9 \%$ in $2008^{2}$, and there were 30,892 new TB cases in 2016, of which 852 cases were MDR-TB ${ }^{3}$.

It is important to quickly identify the results of drug susceptibility testing (DST) in patients at increased risk of drug resistance and to choose individualized treatment regimens based on DST profiles. Korean guidelines for TB, issued in 
2017, recommend that genotypic RIF resistance should be treated as MDR-TB until conventional DST results are identified $^{4}$. Early detection of drug resistance allows immediate and effective treatment, which improves the prognosis and prevents MDR strains from transmission as well as acquisition of additional resistance. Although conventional DST using liquid media culture has shortened the time to 2 weeks compared to solid media culture using Löwenstein-Jensen (LJ) medium, recent advances in molecular tools, such as the detection of mutations associated with drug resistance, have helped to detect drug resistance earlier. Based on this, the WHO recommended the use of molecular line probe assays (LPAs) for rapid detection of MDR-TB in $2016^{5}$.

The GenoType MTBDRplus assay (MTBDRplus assay; Hain Lifescience, Nehren, Germany) is a rapid molecular diagnostic test that simultaneously detects the presence or absence of Mycobacterium tuberculosis and mutations causing resistance to specific drugs ${ }^{6}$. This assay is one of the commercial versions of LPAs that detect drug resistance to RIF and INH relying on specific probes immobilized on nitrocellulose strips. INH resistance is known to be mostly related with mutations in $k a t G$ and inh $A$ gene $^{7-9}$. Mutations in $k a t G$ have been found to be associated with a high-level INH resistance, while inh $A$ mutations produce both low-level resistance to INH and highlevel resistance to prothionamide (Pto), which is used for the treatment of MDR-TB ${ }^{10-14}$. Cross-resistance to Pto may occur in patients who had received INH but had never been treated with Pto ${ }^{15}$, and the frequency of cross-resistance in Korea has been reported to be $50 \%{ }^{16}$.

Currently, Pto resistance can only be detected by phenotypic susceptibility test; however, the result of this test is often questioned due to low reliability and reproducibility ${ }^{17}$. Therefore, verifying the presence of inh $A$ mutation helps determine the use of Pto in cases of MDR-TB before conventional DST is confirmed. Nevertheless, few studies have reported the correlation between the results of the MTBDRplus assay and phenotypic DST for Pto resistance.

In this study, we sought to analyze the relationship between gene mutation pattern according to the MTBDRplus assay and phenotypic INH and Pto susceptibility by conventional DST in patients with mutations on $k a t G$ or inh $A$ genes.

\section{Materials and Methods}

\section{Study population}

This study retrospectively enrolled patients whose MTBDRplus assay results revealed kat $G$ or inhA gene mutations from September 2009 to October 2016 at a single tertiary referral center, Asan Medical Center, Seoul, Korea. Baseline characteristics at the time of diagnosis were gathered, including age, sex, physical examination findings, body mass index (BMI), and medical history (previous anti-TB treatment, diabetes mellitus, chronic liver disease, chronic kidney disease stage $\geq 2$, respiratory disease, transplantation, human immunodeficiency virus infection, anti-tumor necrosis factor [TNF]- $\alpha$ therapy, and hematologic malignancy). The study was approved by the Institutional Review Board of Asan Medical Center (approval number: 2017-1012). The requirement for informed consent was waived due to the retrospective nature of the study.

\section{Collection of specimens and drug susceptibility test}

Specimens from lung (sputum, bronchial washing by bronchofibroscopy, and lung tissue by percutaneous needle aspiration) and other involved organs (cerebrospinal fluid, intestine, lymph nodes, pleural fluid, kidney, bone, and muscle) were included. All specimens were subjected to Ziehl-Neelsen staining for examination of acid-fast bacilli (AFB) on smear and culture using both solid (Ogawa medium; Korean Institute of Tuberculosis, Cheongju, Korea) and liquid (BACTEC 960 Mycobacterial Growth Indicator Tube; Becton Dickinson, Sparks, MD, USA) media. MTBDRplus assay was performed on AFB-positive specimens or clinical isolates in patients with increased risk of drug resistance. Cultured clinical isolates were subjected to conventional DST. Conventional DST was evaluated by the absolute concentration method with LJ media at the Korean Institute of Tuberculosis, a Supranational TB Reference Laboratory. Growth exceeding that of the control wells by $>1 \%$ was considered to indicate drug resistance. A low-level resistance to INH was defined as resistance to 0.2 $\mu \mathrm{g} / \mathrm{mL}$ but susceptible to $1 \mu \mathrm{g} / \mathrm{mL}$ of INH, while a high-level resistance was defined as resistance to INH of $1 \mu \mathrm{g} / \mathrm{mL}$. Isolates were tested for resistance to RIF using concentrations of $40 \mu \mathrm{g} / \mathrm{mL}$. The MTBDRplus assay was performed according to the manufacturer's instructions. The assay detects the presence or absence of wild-type (WT) and mutant DNA sequences in $r p o B, \operatorname{kat} G$, and $i n h A$, which are known to be associated with RIF and INH resistance. There are 27 reaction lines comprising six control lines and $21 \mathrm{WT}$ or mutation reaction lines (MUT) as follows: eight rpoB WT (codon 505 to 533) and four MUT bands (MUT1, D516V; MUT2A, H526Y; MUT2B, H526D; MUT3, S531L), one katG WT (S315 region), and two MUT bands (MUT1, S315T[AGC315ACC]; MUT2, S315T[AGC315ACA]), two inhA WT (WT1, positions -15 to -16; WT2, positions -8), and four MUT bands (MUT1, C15T; MUT2, A16G; MUT3A, T8C; MUT3B, T8A). A gene mutation indicates the presence of MUT band and/or loss of WT band.

\section{Statistical analysis}

Descriptive statistics were used to describe patient characteristics. Categorical variables were reported as counts and percentages and compared using the Pearson's chi-square test or Fisher exact test. Continuously measured parameters were 
reported as means \pm standard deviation and were compared using student's t-test. Statistical significance was indicated for p-values of $<0.05$. All statistical analyses were completed using SPSS version 21.0 (IBM Corp., Armonk, NY, USA).

\section{Results}

\section{Baseline clinical characteristics}

In this retrospective study, the authors reviewed 206 patients whose MTBDRplus assay results revealed kat $G$ or inh $A$ gene mutations. The most common type of infection was pulmonary TB ( $\mathrm{n}=185,89.8 \%$ ) followed by disseminated TB $(\mathrm{n}=12,5.8 \%)$ and extrapulmonary TB (total $\mathrm{n}=9,4.4 \%$; lymphadenitis, $n=5$; intestine, $n=2$; kidney, $n=1$; osteomyelitis, $n=1$ ). Specimens were obtained from sputum $(\mathrm{n}=185)$, bronchial washing ( $\mathrm{n}=3)$, lung tissue $(\mathrm{n}=1)$, and other involved organs (lymph node, $\mathrm{n}=6$; pleural fluid, $\mathrm{n}=4$; cerebrospinal fluid, $\mathrm{n}=2$; intestine, $\mathrm{n}=2$; renal abscess, $\mathrm{n}=1$; spine, $\mathrm{n}=1$; muscle, $\mathrm{n}=1$ ).

Stratifying by genotypic resistance to RIF, $54.9 \%$ isolates were found to be MDR and $45.1 \%$ were found to be INH mono-resistant by MTBDRplus assay (Table 1). Patients with MDR-TB were younger ( $43.0 \pm 16.1$ vs. $50.9 \pm 16.9$ years, $\mathrm{p}=0.001$ ) and had a more frequent history of previous TB treatment $(53.1 \% \mathrm{vs}$. $20.4 \%, \mathrm{p}<0.001)$ than those with INH mono-resistance. There

Table 1. The baseline characteristics of 206 patients with a genotypic resistance to isoniazid, stratified by the genotypic rifampin resistance

\begin{tabular}{|c|c|c|c|c|}
\hline Variable & INH mono-resistance & MDR-TB & Total & p-value \\
\hline No. of subjects & $93(45.1)$ & $113(54.9)$ & $206(100)$ & \\
\hline Age, yr & $50.9 \pm 16.9$ & $43.0 \pm 16.1$ & $46.6 \pm 17.0$ & 0.001 \\
\hline Male sex & $62(66.7)$ & $70(61.9)$ & $132(64.1)$ & 0.482 \\
\hline $\mathrm{BMI}, \mathrm{kg} / \mathrm{m}^{2}$ & $21.3 \pm 3.4$ & $21.1 \pm 3.0$ & $21.2 \pm 3.2$ & 0.745 \\
\hline Previous TB history & $19(20.4)$ & $60(53.1)$ & $79(38.3)$ & $<0.001$ \\
\hline \multicolumn{5}{|l|}{ Concomitant disease } \\
\hline Diabetes mellitus & $12(12.9)$ & $19(16.8)$ & $31(15.0)$ & 0.435 \\
\hline Chronic liver disease* & $10(10.8)$ & $3(2.7)$ & $13(6.3)$ & 0.017 \\
\hline Chronic kidney disease & $4(4.3)$ & $1(0.9)$ & $5(2.4)$ & 0.177 \\
\hline Respiratory disease $^{+}$ & $7(7.5)$ & $5(4.4)$ & $12(5.8)$ & 0.344 \\
\hline Transplantation & $5(5.4)$ & $2(1.8)$ & $7(3.4)$ & 0.248 \\
\hline Others $^{*}$ & $7(7.5)$ & $5(4.4)$ & $12(5.8)$ & 0.344 \\
\hline Positive HIV test & $1(1.1)$ & $0(0)$ & $1(0.5)$ & 0.451 \\
\hline \multicolumn{5}{|l|}{ Phenotypic DST results } \\
\hline INH-mono resistance & $75(80.6)$ & $6(5.3)$ & $81(39.3)$ & \\
\hline High-level resistance & $54(58.1)$ & $4(3.5)$ & $58(28.2)$ & \\
\hline Low-level resistance & $21(22.6)$ & $2(1.8)$ & $23(11.2)$ & \\
\hline MDR-TB & $2(2.2)$ & $105(92.9)$ & $107(51.9)$ & \\
\hline RIF-mono resistance & $0(0)$ & $2(1.8)$ & $2(1.0)$ & \\
\hline Susceptible to INH/RIF & $16(17.2)$ & $0(0)$ & $16(7.8)$ & \\
\hline
\end{tabular}

Values are presented as number (\%).

*Chronic liver disease included alcoholic LC and chronic hepatitis B and C. ${ }^{\dagger}$ Respiratory disease included lung/tracheal cancer ( $\mathrm{n}=4$ ), COPD $(\mathrm{n}=2)$, and pneumoconiosis $(\mathrm{n}=1)$ in INH-mono resistance, and lung cancer $(\mathrm{n}=3)$, COPD $(\mathrm{n}=1)$, and IPF $(\mathrm{n}=1)$ in MDR-TB. ${ }^{*}$ Other comorbidities in INH-mono resistant TB patients included Crohn's disease ( $\mathrm{n}=2$; one with anti-TNF- $\alpha$ therapy and one without), hematologic malignancy without bone marrow transplantation ( $\mathrm{n}=2$; one with myelodysplastic syndrome and one with lymphoma), adrenal insufficiency $(\mathrm{n}=1)$, c-ANCA associated vasculitis ( $\mathrm{n}=1)$, and IgA nephropathy with normal kidney function $(\mathrm{n}=1)$. MDR-TB patients had rheumatoid arthritis on anti-TNF $\alpha$ therapy $(n=1)$, hematologic malignancy without bone marrow transplantation $(n=2$; one with acute myeloid leukemia and one with lymphoma), and adrenal insufficiency ( $\mathrm{n}=2)$.

INH: isoniazid; MDR-TB: multidrug resistant tuberculosis; BMI: body mass index; TB: tuberculosis; HIV: human immunodeficiency virus; DST: drug sensitivity test; RIF: rifampin; LC: liver cirrhosis; COPD: chronic obstructive pulmonary disease; IPF: idiopathic pulmonary fibrosis; TNF- $\alpha$ : tumor necrosis factor $\alpha$. 
was no difference in sex and BMI. Comorbidities were similar in both groups except for chronic liver disease $(2.7 \%$ in MDRTB vs. $10.8 \%$ in INH mono-resistance, $\mathrm{p}=0.017$ ).

\section{Mutation patterns of $k a t G$ and inh $A$ genes by MTBDRplus assay}

Table 2 shows mutation patterns of $k a t G$ and inh $A$ genes detected by the MTBDRplus assay. kat $G$ mutations were identified in 140 of 206 isolates $(68.0 \%)$ and inhA mutations were identified in 72 of 206 isolates (35.0\%). Except for six patients with both $k a t G$ and inhA mutations, MDR-TB patients had more frequent mutations in $k a t G$ than INH mono-resistant TB patients $(69.9 \%$ vs. $59.1 \%$, $\mathrm{p}=0.107)$, although this difference was not statistically significant. In contrast, MDR-TB patients had significantly less frequent inhA mutations $(25.7 \%$ vs. $39.8 \%, \mathrm{p}=0.031$ ).

3. Correlation between phenotypic and genotypic susceptibility results based on mutation patterns of kat $G$ and inh $A$ genes

The concordances of the MTBDRplus assay and the conventional DST for the detection of INH and Pto susceptibility are shown in Table 3. Strains with isolated katG mutation presented phenotypic INH resistance in $97.0 \%$ and phenotypic Pto resistance in $8.2 \%$ of cases. Among the isolates with isolated inhA mutation, $78.8 \%$ were identified as phenotypic INH resistant and $31.8 \%$ as phenotypic Pto resistant. Of all genotypic INH resistant patients, $2.9 \%$ had mutations in both the kat $G$ and inh $A$ genes, all of which were resistant to INH, and $33.3 \%$ were resistant to Pto. Except for six patients with both kat $G$ and inhA mutations, the presence of $k a t G$ mutation presented high-level INH resistance in most cases (94.8\%) while over half of the patients with inhA mutation (51.5\%) showed low-level resistance. Of the 34 phenotypic Pto resistant isolates, 21 (61.8\%) had an inhA mutation, 11 (32.4\%) had a kat $G$ mutation, and two (5.9\%) had both mutations.

Among the 134 strains with isolated $k a t G$ mutation, MUT1 band was observed in 119 (88.8\%) isolates, and positive MUT2 band and WT loss alone were observed in eight (6.0\%) and seven $(5.2 \%)$, respectively. Among the 66 isolates with isolated inh A mutation, the most common type was MUT1 ( $\mathrm{n}=60$, 91.0\%), followed by WT1 loss alone $(\mathrm{n}=4,6.1 \%)$ and MUT3A $(\mathrm{n}=1,1.5 \%)$, and MUT3B $(\mathrm{n}=1,1.5 \%)$. Among 127 strains with isolated katG mutant band (MUT1 or MUT2), 123 (96.9\%) presented resistance to INH and $11(8.7 \%)$ to Pto. Isolates with $k a t G$ WT loss alone were all high-level INH resistant and Pto susceptible (n=7). Among 62 strains with isolated inhA mutant band (MUT1, MUT2, MUT2A, or MUT3B), 51 (82.3\%) were INH resistant and 21 (33.9\%) were Pto resistant. inhA WT loss alone was found in four isolates, of which one (25\%) showed high-level INH-resistance and none showed Pto resistance.

\section{Discussion}

This study analyzed the relationship between gene mutation pattern and phenotypic INH and Pto resistance using the

Table 2. The mutation patterns of the $k a t G$ and $i n h A$ genes in 206 patients with a genotypic INH resistance

\begin{tabular}{|c|c|c|c|c|}
\hline Group & INH mono-resistance & MDR-TB & Total & p-value \\
\hline No. of subjects & $93(45.1)$ & $113(54.9)$ & $206(100)$ & \\
\hline kat $G$ mutation alone & $55(59.1)$ & $79(69.9)$ & $134(65.0)$ & 0.107 \\
\hline Mutant probe $(+)$ & $50(53.8)$ & $77(68.1)$ & $127(61.7)$ & 0.035 \\
\hline Wild-type band loss alone & $5(5.4)$ & $2(1.8)$ & $7(3.4)$ & 0.248 \\
\hline inhA mutation alone (\%) & $37(39.8)$ & $29(25.7)$ & $66(32.0)$ & 0.031 \\
\hline Mutant probe (+) & $33(35.5)$ & $29(25.7)$ & $62(30.1)$ & 0.126 \\
\hline Wild-type band loss alone & $4(4.3)$ & $0(0)$ & $4(1.9)$ & 0.040 \\
\hline Both gene mutation & $1(1.1)$ & $5(4.4)$ & $6(2.9)$ & 0.225 \\
\hline $\operatorname{katG}$ (MUT1), inhA (MUT1) & $0(0)$ & $2(1.8)$ & $2(1.0)$ & \\
\hline katG (MUT1), inhA (WT1 loss) & $1(1.1)$ & $0(0)$ & $1(0.5)$ & \\
\hline katG (MUT1), inhA (WT2 loss) & $0(0)$ & $1(0.9)$ & $1(0.5)$ & \\
\hline katG (WT loss),inhA (MUT1) & $0(0)$ & $1(0.9)$ & $1(0.5)$ & \\
\hline katG (WT loss), inhA (WT2 loss) & $0(0)$ & $1(0.9)$ & $1(0.5)$ & \\
\hline
\end{tabular}

Values are presented as number (\%).

INH: isoniazid; MDR-TB: multidrug-resistant tuberculosis; katG WT: S315 region; katG MUT1: S315T[AGC315ACC]; inhA WT1: positions -15 to -16; inhA WT2: positions -8; inhA MUT1: C15T. 
Table 3. The phenotypic susceptibility to INH and Pto based on the specific mutation patterns of $k a t G$ and inh $A$

\begin{tabular}{|c|c|c|c|c|c|}
\hline \multirow{2}{*}{ Mutation } & \multirow{2}{*}{ Total } & \multicolumn{3}{|c|}{ Phenotypic INH resistance } & \multirow{2}{*}{$\begin{array}{l}\text { Phenotypic Pto } \\
\text { resistance }\end{array}$} \\
\hline & & High-level & Low-level & Total & \\
\hline No. of subjects & $206(100)$ & $149(72.3)$ & 39 (18.9) & $188(91.3)$ & $34(16.5)$ \\
\hline kat $G$ gene alone & $134(65.0)$ & $127(94.8)$ & $3(2.2)$ & $130(97.0)$ & $11(8.2)$ \\
\hline MUT1 (+) & $119(57.8)$ & $112(94.1)$ & $3(2.5)$ & $115(96.6)$ & $9(7.6)$ \\
\hline MUT2 (+) & $8(3.9)$ & $8(100)$ & $0(0)$ & $8(100)$ & $2(25)$ \\
\hline WT (-)/MUT1 (-)/MUT2 (-) & $7(3.4)$ & $7(100)$ & $0(0)$ & $7(100)$ & $0(0)$ \\
\hline inhA gene alone & $66(32.0)$ & $18(27.3)$ & $34(51.5)$ & $52(78.8)$ & $21(31.8)$ \\
\hline MUT1 (+) & $60(29.1)$ & $17(28.3)$ & $34(56.7)$ & $51(85)$ & $21(35)$ \\
\hline MUT2 (+) & $0(0)$ & $0(0)$ & $0(0)$ & $0(0)$ & $0(0)$ \\
\hline $\operatorname{MUT3A}(+)$ & $1(0.5)$ & $0(0)$ & $0(0)$ & $0(0)$ & $0(0)$ \\
\hline MUT3B (+) & $1(0.5)$ & $0(0)$ & $0(0)$ & $0(0)$ & $0(0)$ \\
\hline WT1 (-) and all MUT (-) & $4(1.9)$ & $1(25)$ & $0(0)$ & $1(25)$ & $0(0)$ \\
\hline WT2 (-) and all MUT (-) & $0(0)$ & $0(0)$ & $0(0)$ & $0(0)$ & $0(0)$ \\
\hline Both gene mutation & $6(2.9)$ & $4(66.7)$ & $2(33.3)$ & $6(100)$ & $2(33.3)$ \\
\hline katG (MUT1),inhA (MUT1) & $2(1.0)$ & $2(100)$ & $0(0)$ & $2(100)$ & $0(0)$ \\
\hline katG (MUT1),inhA (WT1 loss) & $1(0.5)$ & $0(0)$ & $1(100)$ & $1(100)$ & $0(0)$ \\
\hline katG (MUT1),inhA (WT2 loss) & $1(0.5)$ & $0(0)$ & $1(100)$ & $1(100)$ & $0(0)$ \\
\hline katG (WT loss),inhA (MUT1) & $1(0.5)$ & $1(100)$ & $0(0)$ & $1(100)$ & $1(100)$ \\
\hline katG (WT loss), inhA (WT2 loss) & $1(0.5)$ & $1(100)$ & $0(0)$ & $1(100)$ & $1(100)$ \\
\hline
\end{tabular}

Values are presented as number (\%).

INH: isoniazid; Pto: prothionamide; katG WT: S315 region; katG MUT1, S315T[AGC315ACC]; katG MUT2, S315T[AGC315ACA]); inhA WT1: positions -15 to -16; inhA WT2: positions -8; inhA MUT1: C15T; inhA MUT2, A16G; inhA MUT3A: T8C; inhA MUT3B: T8A.

MTBDRplus assay. We analyzed 206 genotypic INH-resistant strains and found that $68.0 \%$ had a kat $G$ mutation and $35.0 \%$ had an inhA mutation. The presence of $k a t G$ mutation presented high-level INH resistance in most case (94.8\%), while over half of the patients with inhA mutation (51.5\%) showed low-level resistance, which is consistent with previous studies $^{10-12}$. However, only $31.8 \%$ showed phenotypic Pto resistance in 66 patients with inhA mutations.

In our study, $k a t G$ mutations were more frequent in MDRTB isolates, and inhA mutations were significantly more frequent in INH mono-resistant isolates. According to previous studies, the frequency of S315T substitution in the kat $G$ gene is higher in MDR-TB than in INH mono-resistant $\mathrm{TB}^{18,19}$ and might be related to the higher transmission capabilities of MDR-TB $^{20}$.

Among the isolates with $k a t G$ mutation, MUT1 (S315T) was the most common mutation. This is a transitional mutation $\mathrm{G} \rightarrow \mathrm{C}$ (AGC $\rightarrow \mathrm{ACC}$ ), resulting in the substitution of serine by threonine $(\mathrm{S} \rightarrow \mathrm{T})$ in the amino acid chain. This S315T mutation has been found in up to $94 \%$ of the INH-resistant $\mathrm{TB}^{21}$, which is in agreement with our results. Among the isolates with inhA mutation, MUT1 $(-15 \mathrm{C} \rightarrow \mathrm{T})$ in the inh $A$ promoter was the most common mutation. This mutation is found in up to $35 \%$ of INH-resistant and $55 \%$ of Pto-resistant $\mathrm{TB}^{22}$, which is also in agreement with our results.

Phenotypic Pto resistance presented in $8.2 \%$ of isolated kat $G$ mutations and $31.8 \%$ of isolated inhA mutations. In phenotypic Pto resistant isolates, $61.8 \%$ had inhA mutation, which agrees with the results of previous studies. Vilcheze and $J^{2} \operatorname{cobs}^{22}$ mentioned that the proportion of inhA mutations in Pto-resistant TB is $68 \%$ on average, suggesting that this is the main mechanism of Pto resistance in M. tuberculosis. Pto is a structural analog of INH used as a second-line drug in the treatment of MDR-TB. Both INH and Pto are classified as pro-drugs that need to be activated by different mycobacterial enzymes. INH is activated by the kat $G$-encoded catalaseperoxidase $^{23}$, and Pto is activated by the NADPH-specific flavin adeninedinucleotide-containing monooxygenase EthA, which is regulated by $\mathrm{EthR}^{24}$. Once activated, the mode of action of each drug is very similar such that the active forms of the drugs react with $\mathrm{NAD}^{+}$to yield an INH-NAD or ETH-NAD adduct, which inhibits inhA, leading to disruption of mycolic 
acid biosynthesis and cell death ${ }^{22}$. Therefore, mutations at the inhA gene can cause cross-resistance to INH and Pto by resulting in the overexpression or modification of the inh $A$ target ${ }^{25,26}$. In addition to the inh $A$ mutation, $n d h$ mutation causes cross-resistance to both drugs ${ }^{27,28}$, and ethA, ethR, or $m s h A$ gene mutation also results in Pto mutation ${ }^{22}$. Because inhA mutation is considered as the major mechanism of Pto resistance in TB as previously mentioned, the use of LPAs would help physicians to get information about the use of Pto as well as RIF and INH. Nevertheless, few studies have reported the correlation between the results of the MTBDRplus assay and phenotypic DST for Pto resistance. Machado et al. ${ }^{25}$ examined 17 MDR-TB isolates and found that the presence of a mutation in the inhA regulatory region together with a mutation in the inh $A$ coding region can lead to the development of high-level INH resistance and cross-resistance to Pto. Niehaus et al. ${ }^{29}$ sought to determine the proportion of patients who could potentially benefit from high-dose INH and who may be resistant to Pto. However, phenotypic Pto resistance was deduced from the inhA mutation because phenotypic DST was not performed. Rueda et al. ${ }^{30}$ examined the relationship between mutations and phenotypic resistance; however, they used whole genome sequencing, which is generally difficult for widespread use in clinical fields. In this study, only $31.8 \%$ showed phenotypic Pto resistance in 66 patients with inhA mutations, suggesting that Pto may be selected as one of MDR-TB regimen in patients with inhA mutation detected by MTBDRplus assay until phenotypic DST confirms Pto resistance. There could be two main speculations for the discrepancy between our hypothesis and the results. First, the low reliability and reproducibility of conventional DST of Pto might be the cause of the discrepant results ${ }^{17}$. Secondly, other compensatory mechanisms might play a role in maintaining Pto susceptibility in $M$. tuberculosis strains. Tan et al. ${ }^{31}$ found that mutations in the promoter region of inhA and inhA gene in Pto-susceptible isolates were different from those in Pto-resistant isolates, and these mutations are more likely to appear together with other mutations in Pto-resistant isolates. They suggested that these mutations may show cumulative effect on increasing minimum inhibitory concentration values of $M$. tuberculosis against Pto. We also speculate that mutations in specific parts of inhA may not affect Pto susceptibility.

Although the number of cases in this study was small, isolates with $k a t G$ WT loss alone were all high-level INH resistant, while isolates with inhA WT loss alone were INHresistant in only $25 \%$ of cases. Han et al..$^{32}$ found one of 144 isolates showed low-level INH resistance with ahpC mutation confirmed by other LPA (AdvanSure MDR-TB GenoBlot assay), which revealed susceptibility to INH by conventional DST. The authors interpreted that the discrepancy might result from false positive of LPA, low-level resistance suppressing growth of $M$. tuberculosis at $0.2 \mu \mathrm{g} / \mathrm{mL}$ of INH, or heteroresistance in which susceptible and resistant strains coexist.
Therefore, in the case of genotypic INH resistance with isolated inhA wild type loss, we recommend that the possibility of an INH susceptible strain should not be ruled out before the final phenotypic DST results are obtained. None of four patients with loss of WT inhA band alone showed Pto resistance. Due to the small number of subjects and the absence of gene sequencing data, we could not draw any conclusion from this data. Further studies are needed to reveal the real meaning of this finding.

This study has several limitations. In patients with discrepant results between conventional and molecular DST, gene sequencing, a gold standard method of molecular test, was not performed. The phenotypic DST of second-line drugs such as Pto is considered as an unreliable test, but this test, as a gold standard method, was compared to MTBDRplus assay results. Finally, even though we enrolled a large number of isolates with $k a t G$ or inh $A$ mutations, the number of isolates with inh $A$ WT loss alone was too small to draw any firm conclusion.

The kat $G$ and inhA mutations detected by MTBDRplus assay are good indicators of phenotypic INH resistance. However, only about one-third of inhA mutations revealed phenotypic Pto resistance, suggesting that Pto may be selected as one of MDR-TB regimen in patients with inhA mutation detected by MTBDRplus assay until conventional DST confirms phenotypic Pto resistance. We suggest that the reporting of detailed mutation patterns of MTBDRplus assay would be important for clinical practice, rather than simply presenting the resistance or susceptibility results.

\section{Authors' Contributions}

Conceptualization: Shim TS. Methodology: Shim TS. Formal analysis: Lee JH, Jo KW. Data curation: Shim TS. Software: Lee JH. Validation: Jo KW, Shim TS. Investigation: Lee JH. Writing original draft preparation: Lee JH. Writing - review and editing: Jo KW, Shim TS. Approval of final manuscript: all authors.

\section{Conflicts of Interest}

No potential conflict of interest relevant to this article was reported.

\section{References}

1. World Health Organization. Global tuberculosis report 2016 WHO/HTM/TB/2016.13. Geneva: World Health Organization; 2016.

2. Bai GH, Park YK, Choi YW, Bai JI, Kim HJ, Chang CL, et al. Trend of anti-tuberculosis drug resistance in Korea, 19942004. Int J Tuberc Lung Dis 2007;1 1:571-6. 
3. Korea Centers for Disease Control and Prevention. Annual report on the notified tuberculosis in Korea, 2016. Cheongju: Korea Centers for Disease Control and Prevention; 2016.

4. Korea Centers for Disease Control and Prevention. Korean guidelines for tuberculosis third edition, 2017. Cheongju: Korea Centers for Disease Control and Prevention; 2017.

5. World Health Organization. WHO treatment guidelines for drug-resistant tuberculosis, 2016 update. WHO/HTM/ TB/2016.04. Geneva: World Health Organization; 2016.

6. Leylabadlo HE, Kafil HS, Yousefi M, Aghazadeh M, Asgharzadeh M. Pulmonary tuberculosis diagnosis: where we are? Tuberc Respir Dis 2016;79:134-42.

7. Rouse DA, Li Z, Bai GH, Morris SL. Characterization of the $k a t G$ and $i n h A$ genes of isoniazid-resistant clinical isolates of Mycobacterium tuberculosis. Antimicrob Agents Chemother 1995;39:2472-7.

8. Guo H, Seet Q, Denkin S, Parsons L, Zhang Y. Molecular characterization of isoniazid-resistant clinical isolates of Mycobacterium tuberculosis from the USA. J Med Microbiol 2006;55(Pt 11):1527-31.

9. Seifert M, Catanzaro D, Catanzaro A, Rodwell TC. Genetic mutations associated with isoniazid resistance in Mycobacterium tuberculosis: a systematic review. PLoS One 2015; 10:e0119628.

10. Brossier F, Veziris N, Truffot-Pernot C, Jarlier V, Sougakoff W. Performance of the genotype MTBDR line probe assay for detection of resistance to rifampin and isoniazid in strains of Mycobacterium tuberculosis with low- and high-level resistance. J Clin Microbiol 2006;44:3659-64.

11. Gali N, Dominguez J, Blanco S, Prat C, Alcaide F, Coll P, et al. Use of a mycobacteriophage-based assay for rapid assessment of susceptibilities of Mycobacterium tuberculosis isolates to isoniazid and influence of resistance level on assay performance. J Clin Microbiol 2006;44:201-5.

12. Lavender C, Globan M, Sievers A, Billman-Jacobe H, Fyfe J. Molecular characterization of isoniazid-resistant Mycobacterium tuberculosis isolates collected in Australia. Antimicrob Agents Chemother 2005;49:4068-74.

13. Morlock GP, Metchock B, Sikes D, Crawford JT, Cooksey RC. ethA, inhA, and katG loci of ethionamide-resistant clinical Mycobacterium tuberculosis isolates. Antimicrob Agents Chemother 2003;47:3799-805.

14. Engohang-Ndong J, Baillat D, Aumercier M, Bellefontaine F, Besra GS, Locht C, et al. EthR, a repressor of the TetR/CamR family implicated in ethionamide resistance in mycobacteria, octamerizes cooperatively on its operator. Mol Microbiol 2004;51:175-88.

15. Banerjee A, Dubnau E, Quemard A, Balasubramanian V, Um $\mathrm{KS}$, Wilson T, et al. inhA, a gene encoding a target for isoniazid and ethionamide in Mycobacterium tuberculosis. Science 1994;263:227-30.

16. Lee H, Cho SN, Bang HE, Lee JH, Bai GH, Kim SJ, et al. Exclusive mutations related to isoniazid and ethionamide resistance among Mycobacterium tuberculosis isolates from Korea. Int J Tuberc Lung Dis 2000;4:441-7.

17. Lin SY, Desmond E, Bonato D, Gross W, Siddiqi S. Multicenter evaluation of Bactec MGIT 960 system for second-line drug susceptibility testing of Mycobacterium tuberculosis complex. J Clin Microbiol 2009;47:3630-4.

18. Unissa AN, Selvakumar N, Narayanan S, Suganthi C, Hanna LE. Investigation of Ser315 substitutions within katG gene in isoniazid-resistant clinical isolates of Mycobacterium tuberculosis from south India. Biomed Res Int 2015;2015:257983.

19. van Doorn HR, de Haas PE, Kremer K, VandenbrouckeGrauls CM, Borgdorff MW, van Soolingen D. Public health impact of isoniazid-resistant Mycobacterium tuberculosis strains with a mutation at amino-acid position 315 of $k a t G$ : a decade of experience in The Netherlands. Clin Microbiol Infect 2006;12:769-75.

20. Gagneux S, Burgos MV, DeRiemer K, Encisco A, Munoz S, Hopewell PC, et al. Impact of bacterial genetics on the transmission of isoniazid-resistant Mycobacterium tuberculosis. PLoS Pathog 2006;2:e61.

21. Mokrousov I, Narvskaya O, Otten T, Limeschenko E, Steklova L, Vyshnevskiy B. High prevalence of KatG Ser315Thr substitution among isoniazid-resistant Mycobacterium tuberculosis clinical isolates from northwestern Russia, 1996 to 2001. Antimicrob Agents Chemother 2002;46:1417-24.

22. Vilcheze C, Jacobs WR Jr. Resistance to isoniazid and ethionamide in Mycobacterium tuberculosis: genes, mutations, and causalities. Microbiol Spectr 2014;2:MGM2-0014-2013.

23. Lei B, Wei CJ, Tu SC. Action mechanism of antitubercular isoniazid: activation by Mycobacterium tuberculosis KatG, isolation, and characterization of inha inhibitor. J Biol Chem 2000;275:2520-6.

24. Vannelli TA, Dykman A, Ortiz de Montellano PR. The antituberculosis drug ethionamide is activated by a flavoprotein monooxygenase. J Biol Chem 2002;277:12824-9.

25. Machado D, Perdigao J, Ramos J, Couto I, Portugal I, Ritter C, et al. High-level resistance to isoniazid and ethionamide in multidrug-resistant Mycobacterium tuberculosis of the Lisboa family is associated with inhA double mutations. J Antimicrob Chemother 2013;68:1728-32.

26. Larsen MH, Vilcheze C, Kremer L, Besra GS, Parsons L, Salfinger M, et al. Overexpression of inhA, but not kasA, confers resistance to isoniazid and ethionamide in Mycobacterium smegmatis, M. bovis BCG and M. tuberculosis. Mol Microbiol 2002;46:453-66.

27. Vilcheze C, Weisbrod TR, Chen B, Kremer L, Hazbon MH, Wang F, et al. Altered NADH/NAD+ ratio mediates coresistance to isoniazid and ethionamide in mycobacteria. Antimicrob Agents Chemother 2005;49:708-20.

28. Cardoso RF, Cardoso MA, Leite CQ, Sato DN, Mamizuka EM, Hirata RD, et al. Characterization of ndh gene of isoniazid resistant and susceptible Mycobacterium tuberculosis isolates from Brazil. Mem Inst Oswaldo Cruz 2007;102:59-61. 
29. Niehaus AJ, Mlisana K, Gandhi NR, Mathema B, Brust JC. High prevalence of inhA promoter mutations among patients with drug-resistant tuberculosis in KwaZulu-Natal, South Africa. PLoS One 2015;10:e0135003.

30. Rueda J, Realpe T, Mejia GI, Zapata E, Rozo JC, Ferro BE, et al. Genotypic analysis of genes associated with independent resistance and cross-resistance to isoniazid and ethionamide in Mycobacterium tuberculosis clinical isolates. Antimicrob
Agents Chemother 2015;59:7805-10.

31. Tan Y, Su B, Zheng H, Song Y, Wang Y, Pang Y. Molecular characterization of prothionamide-resistant Mycobacterium tuberculosis isolates in southern China. Front Microbiol 2017;8:2358.

32. Han SB, Jo Y, Yu JK, Kim Y, Park YJ. Performance assessment of Advansure MDR-TB Genoblot Assay Kit for anti-tuberculosis drug susceptibility test. Lab Med Online 2012;2:34-40. 